\title{
SIERPINSKI ON THE CONTINUUM
}

Hypothèse du Continu. By W. Sierpinski. (Monografje Matematyczne, Tome 4.) Warsaw, Seminarjum Matematyczne Uniwersytetu Warszawskiego, 1934. v+192 pp.

This book is the fourth of the excellent group of monographs which are appearing under the auspices of the Mathematical Seminar of the University of Warsaw. In it Professor Sierpinski makes an exhaustive study of Cantor's hypothesis of the continuum, which may be stated in an elementary form as the hypothesis that every non-denumerable set of real numbers has the power of the continuum. The form of the hypothesis of the continuum the author most generally uses is that of the equality $2 \boldsymbol{N}_{0}=\boldsymbol{N}_{1}$. He points out that the known demonstrations of the equivalence of these two forms of the hypothesis require the use of the axiom of choice. He distinguishes between the hypothesis of the continuum and the problem of the continuum which is that of finding the place of the continuum amongst the alephs, that is, the problem of determining the ordinal $\alpha$ such that $2 \boldsymbol{N}_{0}=\boldsymbol{N}_{\alpha}$. If the hypothesis of the continuum were true one would have $\alpha=1$. That $\alpha$ is not $\omega$ is a result due to J. König; Sierpinski points out that König's demonstration may be modified so as not to use the axiom of choice.

The author calls attention to the fact that important simplifications would be made in point set theory were one able to demonstrate the hypothesis of the continuum. Many important propositions of different branches of mathematics cannot be deduced at present without using this hypothesis. For persons not recognizing the validity of reasoning based on this hypothesis, it is of extreme importance to know the propositions in whose proof it is used and which we actually do not know how to prove without it.

In Chapter 1 there are given eleven propositions each of which is equivalent to the Hypothesis $\mathrm{H}$. Among those which are the most interesting to the reviewer and also most frequently used in the remainder are the following: $P_{1}$, which states that the plane is the sum of two sets, one of which is at most denumerable on every parallel to the axis of ordinates, and the other at most denumerable on every parallel to the axis of abscissas; $P_{3}$, to the effect that there exists an infinite sequence of single-valued functions of $x, f_{1}(x), f_{2}(x), \cdots$ such that given any non-denumerable set $N$ of real numbers, all functions of the sequence, save possibly a finite number, transform $N$ into the set of all real numbers; $P_{7}$, which states that there exists an analytic linear set which is not the sum of less than $2 \boldsymbol{N}_{0}$ sets measurable $(B)$.

In Chapter 2 the author is concerned mainly with the sets of Lusin, that is, sets of the power of the continuum which have at most a countable set in common with each linear non-dense perfect set. Lusin demonstrated with the aid of the hypothesis of the continuum that these sets, which we shall call sets $L$, exist. Among the interesting properties of these sets that are pointed out are: (1) Every non-denumerable subset is of second category of Baire; (2) Every function of Baire defined on it is of class $\leqq 2$ on the set; (3) Every function of Baire transforms a set with property $L$ into one with property $C$, 
that is, such that given any preassigned sequence $a_{1}, a_{2}, a_{3}, \cdots$ of real positive numbers, it is possible to cover the sets with intervals $I_{1}, I_{2}, I_{3}, \cdots$, where length of $I_{n}=a_{n}$. On a linear non-denumerable set $Q$ with property $L$ there are given examples (1) of a function $f(x)$ continuous on $Q$ but not uniformly continuous on any non-denumerable subset, (2) of an infinite sequence $f_{1}(x), f_{2}(x), \cdots$ of continuous functions which converge non-uniformly toward zero on every non-denumerable subset of $Q$.

An immediate consequence of property (3) of the sets of Lusin is that there exists a linear set of the power of the continuum of which every continuous image is of measure zero. The author also discusses the relation of sets $L$ to sets always of the first category, that is, sets which are of the first category of Baire on every linear perfect set.

In Chapter 3 there is discussed a group of questions dealing with a type of duality between sets of measure zero and sets of the first category. This duality results from a remarkable theorem the proof of which requires the use of Hypothesis $\mathrm{H}:-$

There exists a function $f(x)$ with single-valued inverse defined on the set $\mathcal{E}$ of all real numbers such that $f(\varepsilon)=\mathcal{E}$ and which transforms each set $E \subset \mathcal{E}$ of the first category into a set $f(E)$ of measure zero while its inverse function $f^{-1}(x)$ transforms every set $E \subset \mathcal{E}$ of measure zero into a set $f^{-1}(E)$ of the first category.

Using this duality from the sets of Lusin of the previous chapter, we now get the sets enjoying property $S$, that is, sets which admit with every linear set of measure zero only a denumerable infinity of common points. If $E$ is a linear non-denumerable set with property $S$, then (1) $E$ is non-measurable as is each of its non-denumerable subsets, and (2) every function of Baire defined on $E$ is of class $\leqq 1$ on $E$. Using the duality that has been established, the author now gives a number of theorems concerning the existence of sets such as sets at the same time non-measurable and always of first category, and non-measurable sets enjoying the property of Baire. He then introduces sets with the property $\lambda$, that is, such that each denumerable subset of the given set $E$ is a $G_{\delta}$ relative to $E$. With the aid of the duality established before he shows the existence of linear sets of the power of the continuum with property $\lambda$. According to a theorem by Lusin, there are linear sets always of the first category which do not have property $\lambda$.

In Chapter 4 the author considers a number of other interesting consequences of the hypothesis of the continuum. Extremely interesting is proposition $C_{53}$ which constitutes a negative solution of the problem of generalized measure in that it is proved that, if $Q$ is any set of real numbers, there does not exist any function $m(E)$ which assigns to every subset $E$ of $Q$ a real non-negative number such that

(1) $m(E)$ is not identically zero for all subsets $E$ of $Q$,

(2) $m\left(\sum_{1}^{\infty} E_{n}\right)=\sum_{1}^{\infty} m\left(E_{n}\right)$ whenever the sets $E_{1}, E_{2}, \cdots$ are mutually exclusive subsets of $Q$,

(3) $m(E)=0$ for every subset consisting only of a single element. 
There is also given the theorem of Ulam to the effect that every linear set of positive exterior measure contains a non-denumerable infinity of mutually exclusive sets of positive exterior measure. Other interesting consequences pointed out are: (1) the theorem that every linear set which is everywhere of second category is the sum of a non-denumerable infinity of mutually exclusive sets which are also everywhere of second category, (2)the existence of a function of a real variable which is discontinuous on every non-denumerable set, (3) the existence of a linear non-denumerable set $K$ of the first category such that each translation along the line transforms the set into itself with the exception of at most a denumerable infinity of points. Interesting application is also made to the types of dimensions of Fréchet and analytic sets.

In Chapter 5 the author discusses inaccessible alephs. Ignoring the question of the existence of inaccessible alephs, he shows that there does not exist any inaccessible aleph which does not exceed $2 \aleph_{0}$. He then deduces from this certain important propositions in connection with a property $U$.

In Chapter 6 the author discusses effective examples and the hypothesis of the continuum where one says that a theorem of existence is demonstrated in an effective manner whenever one has defined an individual $a$ and demonstrated that $a$ satisfies the theorem considered. He points out that most examples where we do not know how to demonstrate existence without the use of $\mathrm{H}$ are non-effective. However there are some effective examples of sets enjoying certain properties which do use the hypothesis. He gives as an instance that, by admitting the hypothesis of the continuum, one is able to define effectively an ordered set of power greater than $2 N_{0}$ formed of functions of a real variable.

In Chapter 7 the author discusses several generalized hypotheses of the continuum. Two equivalent ones of these hypotheses are: $G$ to the effect that given any cardinal number $m$ greater than or equal to $\aleph_{0}$, there does not exist any cardinal number $n$ such that $m<n<2^{m}$, and $G^{*}$ which states that, given any ordinal, one has

$$
\aleph_{\alpha+1}=2 \aleph_{\alpha}
$$

In proving this equivalence the axiom of choice is used.

The book furnishes a very interesting analysis of the work that has been done on the consequences of this important hypothesis. In it there is gathered much of the fundamental work of the author, Lusin, Tarski, Kuratowski, and others of this school. One sees how deeply this postulate cuts through all phases of the foundations of mathematics, how intimately many fundamental questions of analysis and geometry are connected with it. Every student of questions in the foundations of our subject will most cordially welcome this very valuable analysis. It is a most excellent addition to our mathematical literature and another one of the fine contributions which the Polish group of mathematicians is making to progress in science.

J. R. KLINE 